\title{
Polyvalent Multifunctional Nanoparticles: A Powerful Tool to Address Various Biomedical Challenges
}

\author{
Yuan Guo ${ }^{1}$ and Dejian Zhou ${ }^{2, *}$ \\ ${ }^{1}$ School of Food Science and Nutrition, and Astbury Centre for Structural Molecular Biology, University of Leeds, Leeds \\ LS2 9JT, U.K. Email: y.guo@leeds.ac.uk. \\ ${ }^{2}$ School of Chemistry and Astbury Centre for Structural Molecular Biology, University of Leeds, Leeds LS2 9JT, U.K. \\ Email: d.zhou@leeds.ac.uk.
}

\section{Extended Abstract}

A great healthcare challenge facing the society is to find solution to some of the most devastating diseases, such as viral infection, neurodegenerative diseases and cancer. We are pursuing a polyvalent multifunctional nanoparticle (PMN) strategy to exploit multivalency (greatly enhanced affinity and specificity), surface chemistry (maximising ligand accessibility and function) and integrate nanoparticles' unique chemical-physical properties to achieve multi-functionality in order to address this challenge. Herein I will introduce two aspects of our research.

(1) PMN-cancer nanomedicine. Owing to excellent biocompatibility, water-solubility, non-cytotoxicity, and universal high cell uptake, polyvalent DNA-gold nanoparticle conjugates (DNA-GNPs) are powerful probes for biomedical research.[1] However, lacking stimuli-response release and limited resistance against nuclease degradation and non-specific adsorption have significantly limited their real-world applications. To overcome such problems, we have introduced an $i$ motif DNA sequence which can produce rapid, $\mathrm{pH}$-dependent conformational switches between single- and four- stranded structures [2] into DNA-GNP, and successfully achieved efficient intracellular acidic pH-triggered drug release inside cancer cells, leading to high anticancer efficacy.[3] We have developed a new PEGylation strategy by hybridizing terminally PEGylated complementary DNA strand onto DNA-GNP, affording it complete resistance against non-specific adsorption and greatly enhanced stability against nuclease degradation without compromising its DNA loading and cell uptake efficiency.[4] Furthermore, we have combined the $i$-motif DNA sequence and gold nanorod to harness their $\mathrm{pH}-$ responsiveness and near-infrared (NIR) photothermal effects, allowing us to achieve $\mathrm{pH} / \mathrm{NIR}$ light dual-responsive drug release inside cancer cells and effectively overcome cancer multidrug resistance at the cellular level.[5]

(2) Glycan-PMN Probes. Multivalent lectin-glycan interactions (LGIs) are widespread and vital for viral infection.[6] Understanding the underlying structural mechanisms is key to develop glycoconjugates that can specifically block such LGIs to prevent infection. Unfortunately, the structures of two vitally important tetrameric lectins, DC-SIGN [7] and DC-SIGNR, which play a key role in facilitating the HIV, HCV, and Ebola virus infections, remain unknown, limiting our ability to design spatial-matched polyvalent glycans to target DC-SIGN/R specifically for therapeutic interventions. To address this challenge, we have pioneered the use of glycan-PMNs as structural probes for LGIs. We have developed a new glycosylation method using lipoic acid-PEG-glycan ligands which works robustly for both quantum dots (QDs) and GNPs.[8-10] By exploiting nanoparticles' unique fluorescence properties, we have developed two new LGI affinity quantification methods, via fluorescence resonance energy transfer with QDs [8,9] or fluorescence quenching with GNPs [10], revealing that glycanPMNs can offer greatly enhanced binding affinity with DC-SIGN (e.g. $\sim 1.5$ million-fold) over the corresponding monovalent binding. Moreover, we show that glycan-PMNs can offer unprecedented $>100$-fold discrimination between DC-SIGN and DC-SIGNR, two-closely tetrameric lectins which were thought to be identical. We further discover that a subtle difference in binding site orientation afford DC-SIGN/R with distinct glycan-PMNs binding modes (simultaneous binding and intercrosslinking for DC-SIGN and DC-SIGNR, respectively), resulting in glycan-PMNs only potently blocking DC-SIGN-, but not DC-SIGNR- mediated virus infection [10]. Finally, we show glycan-GNPs potently inhibit DC-SIGN mediated pseudoEbola virus infection of host cells $\left(\mathrm{IC}_{50} \sim 95 \mathrm{pM}\right.$ ), making it the most potent glycoconjugate inhibitors against DC-SIGNmediated Ebola virus infections.

We thank the UK Wellcome Trust (097354/Z/11/Z) and BBRSC (BB/R007829/1) for funding. We also thank the previous and current Zhou group members (Dr Lei Song, Dr Emma Poole and Dr Darshita Budhadev) and collaborators (Dr Rongjun Chen, Prof. Bruce Turnbull, Dr Nicole Hondow and Prof. Stefan Pöhlmann) for their contribution. 


\section{References}

[1] D. A. Giljohann, D. S. Seferos, W. L. Daniel, M. D. Massich, P. C. Patel, and C. A. Mirkin, "Gold Nanoparticles for Biology and Medicine," Angew. Chem. Int. Ed., vol. 49, no. 19, pp. 3280-3294, 2010.

[2] D. Liu, A. Bruckbauer, C. Abell, S. Balasubramanian, D.J. Kang, D. Klenerman, and D. Zhou, "A reversible pH-driven DNA nanoswitch array," J. Am. Chem. Soc., vol. 128, no. 6, pp. 2067-2071, 2006.

[3] L. Song, V. H. B. Ho, C. Chen, Z. Yang, D. Liu, R. Chen, and D. Zhou, "Efficient, pH-triggered drug delivery using a pH-responsive DNA-conjugated gold nanoparticle," Adv. Healthcare Mater., vol. 2, no. 2, pp. 275-280, 2013.

[4] L. Song, Y. Guo, D. Roebuck, C. Chen, M. Yang, Z. Yang, S. Sreedharan, C. Glover, J. A. Thomas, D. Liu, S. Guo, R. Chen, and D. Zhou, "Terminal PEGylated DNA-gold nanoparticle conjugates offering high resistance to nuclease degradation and efficient intracellular delivery of DNA binding agents," ACS Appl. Mater. Interfaces, vol. 7. No. 33, pp. 18707-18716, 2015.

[5] W. Zhang, F. Wang, Y. Wang, J. Wang, Y. Yu, S. Guo, R. Chen, and D. Zhou, "pH and near-infrared light dual-stimuli responsive drug delivery using DNA-conjugated gold nanorods for effective treatment of multidrug resistant cancer cells," J. Controlled Release, vol. 232, no. 1, pp. 9-19, 2016.

[6] S. Bhatia, L. C. Camacho and R. Haag, "Pathogen Inhibition by Multivalent Ligand Architectures," J. Am. Chem. Soc., vol. 138, no. 42, pp. 8654-8666, 2016.

[7] T. B. Geitenbeek, D. S. Kwon, R. Torensma, S. J. van Vliet, G. C. van Duijnhoven, J. Middel, I. L. Cornelissen, H. S. Nottet, V. N. Kewal-Ramani, D. R. Littman, C. G. Figdor, and Y. van Kooyk, "DC-SIGN, a dendritic cell-specific HIV1-binding protein that enhances trans-infection of T cells," Cell, vol. 100, no. 5, pp. 587-597, 2000.

[8] Y. Guo, C. Sakonsinsiri, I. Nehlmeier, M. A Fascione, H. Zhang, W. Wang, S. Pöhlmann, W. B. Turnbull and D. Zhou, "Compact, polyvalent mannose quantum dots as sensitive, ratiometric FRET probes for multivalent protein-ligand interactions," Angew. Chem. Int. Ed., vol. 55, no. 15, pp. 4738-4742, 2016.

[9] Y. Guo, I. Nehlmeier, E. Poole, C. Sakonsinsiri, N. Hondow, A. Brown, Q. Li, S. Li, J. Whitworth, Z. Li, A. Yu, R. Brydson, W. B. Turnbull, S. Pöhlmann, and D. Zhou, "Dissecting multivalent lectin-carbohydrate recognition using polyvalent multifunctional glycan-quantum dots," J. Am. Chem. Soc., vol. 139, no. 39, pp. 11833-11844, 2017.

[10] D. Budhadev, E. Poole, I. Nehlmeier, Y. Liu, J. Hooper, E. Kalverda, U. S. Akshath, N. Hondow, W. B. Turnbull, S. Pöhlmann, Y. Guo, and D. Zhou, "Glycan-Gold Nanoparticles as Multifunctional Probes for Multivalent LectinCarbohydrate Binding: Implications for Blocking Virus Infection and Nanoparticle Assembly" J. Am. Chem. Soc., vol. 142, no. 42, pp. 18022-18034, 2020. 